\title{
EVALUATION PAR QUESTIONNAIRE DES INCONVENIENTS LIES AUX PROTHESES PENIENNES CHEZ 25 IMPLANTES
}

\author{
P. Kempeneers*, R. Andrianne**, C. Mormont*** \\ *Service de Neuropsychiatrie, CHU de Liège, Domaine Universitaire du Sart Tilman, B-35, 4000 Liège - Belgique \\ **Service d'Urologie, CHU de Liège, Domaine Universitaire du Sart Tilman, B-35, 4000 Liège - Belgique \\ ***Service de Psychologie Clinique, Faculté de Psychologie, Domaine Universitaire du Sart Tilman, B-18, 4000 Liège - Belgique
}

\section{PENILE PROSTHESES : PROBLEMS OF USE IN A SAMPLE OF 25 RECI-} PIENTS. Twenty-five patients who had received a penile prosthesis between 1982 and 1991 responded to a postal follow-up questionnaire. Some men reported pain, urinary problems, loss of penile sensitivity, discomfort and difficulties for manipulating the prosthesis. Nevertheless, many of these problems were minimized by the patients themselves. Moroever, the majority of them seem to decrease with time. In such cases a process of spontaneous functional adaptation was operating. A more delicate problem, which may be partially psychosexual in origin, was complaints concerning the frequency and prolonged duration of erections permitted by the prostheses. In order to reduce such problems, as well as facilitating the functional adaptation, psychosexual support for such patients would be highly advisable. Key-words : Impotence, penile prosthesis. Andrologie 1992, 2 : 130-133.

\section{INTRODUCTION}

L'implantation de prothèses péniennes est devenue une option thérapeutique non négligeable dans le traitement des troubles érectiles .

Entre décembre 1982 et décembre 1991, 44 patients impuissants présentant de sévères affections organiques ont bénéficié de cette solution.

Avec une moyenne de 10 nouveaux implants par an, nous estimons que, dans notre clinique, ce type d'interventions a atteint sa vitesse de croisière en 1988. Il est donc nécessaire que notre équipe interdisciplinaire, le CETISM (Centre d'Etude et de Traitement Interdisciplinaire de Sexopathologie Masculine), se penche aujourd'hui sur son expérience de l'implant pénien afin d'en évaluer les résultats.

Notre projet global d'évaluation ne s'est pas arrêté à la seule analyse des dossiers médicaux des patients implantés. Vingt-cinq d'entre eux ont en effet répondu à un questionnaire de follow-up destiné à saisir quelques-unes des implications de la prothèse dans l'existence quotidienne, et ceci sur les plans psychologique, sexologique et conjugal. Notre objectif n'est pas ici d'en détailler tous les résultats : une seule communication n'y peut suffire. Toutefois, I'enquête réalisée a mis à jour un certain nombre de difficultés spécifiques. Relatifs à la sensibilité du pénis, aux sensations inconfortables, à la manipulation de la prothèse, etc, ces problèmes passaient inaperçus dans les dossiers médicaux . Nous désirons ici en toucher quelques mots.

\section{METHODES ET POPULATION}

En décembre 1991, nous disposions des dossiers de 44 patients implantés .

Leur dépouillement nous a appris que $22,7 \%$ d'entre eux ont présenté des complications bénignes justifiant une ou plusieurs réintervention(s). Pour un tiers, ces problèmes se révélaient d'ordre mécanique: les vices de fonctionnement et, pour les deux autres tiers, d'ordre non-mécanique: les vices d'évolution ainsi que les complications faisant suite au déroulement de l'opération. Trois des 44 patients ont dû être explantés $(6,4 \%)$. La population des usagers actuels d'une prothèse s'élève dès lors à 41 sujets.

Dans le souci de dépasser la seule évaluation médico-technique, un questionnaire de follow-up a été conçu afin d'apprécier l'intégration de la prothèse dans le vécu quotidien des implantés.

Ce questionnaire comporte deux versions. L'une est destinée aux porteurs de prothèse gonflable, I'autre aux porteurs de prothèse semi-rigide.

Chacun des 41 patients actuellement usagers d'une prothèse a reçu, par voie postale, le ques- tionnaire adapté à sa situation: situation relative qui aux modèles gonflables $(\mathrm{N}=29)$, qui aux modèles semi-rigides ( $\mathrm{N}=12)$.

Après rappel, 25 questionnaires nous furent retournés, soit un taux de réponse de $61 \%$. Dixsept de ces questionnaires émanent de porteurs d'un modèle gonflable et huit de porteurs d'une prothèse semi-rigide.

La période de follow-up varie entre un mois et demi et 99 mois, avec une moyenne de 22 mois.

Lors de leur première opération, l'âge moyen des répondants s'établissait à 49 ans et demi, avec des extrêmes de 24 et 64 ans .

\section{PROBLEMES MIS A JOUR PAR L'ENQUE- TE DE FOLLOW-UP}

L'enquête réalisée a donc permis de pointer quelques difficultés rencontrées par les usagers d'une prothèse pénienne . Elles concernent:

- des sensations douloureuses éprouvées lors de la reprise des rapports,

- la sensibilité de la verge émoussée consécutivement à l'intervention,

- des difficultés pour uriner aisément,

- des sensations inconfortables avec certains vêtements,

- des problèmes de manipulation de la prothèse, - des déceptions relatives aux dimensions procurées au pénis par la prothèse.

\section{Sensations douloureuses}

Sur les 25 répondants, un patient signale ressentir de légères douleurs à l'occasion des rapports. Il importe de mentionner que ce patient a

\begin{tabular}{|c|c|c|c|c|c|}
\hline \multirow{2}{*}{$\begin{array}{l}\text { Items extraits du } \\
\text { questionnaire de follow-up }\end{array}$} & \multicolumn{3}{|c|}{ Modalités de réponse } & \multirow{2}{*}{$\mathrm{N}$} & \multirow{2}{*}{$\mathrm{M}$} \\
\hline & \multicolumn{3}{|c|}{ Effectifs } & & \\
\hline \multirow[t]{2}{*}{ Sensibilité émoussée } & $\begin{array}{c}1 \\
\text { non }\end{array}$ & $\begin{array}{c}2 \\
\text { un peu }\end{array}$ & $\begin{array}{c}3 \\
\text { nettement }\end{array}$ & \multirow[t]{2}{*}{24} & \multirow[t]{2}{*}{1,54} \\
\hline & 14 & 7 & 3 & & \\
\hline \multirow{2}{*}{$\begin{array}{l}\text { Appréciation subjective de } \\
\text { la perte de sensibilité }\end{array}$} & $\begin{array}{c}1 \\
\text { peu gênante }\end{array}$ & $\begin{array}{c}2 \\
\text { assez gênante }\end{array}$ & $\begin{array}{c}3 \\
\text { très gênante }\end{array}$ & \multirow[t]{2}{*}{9} & \multirow[t]{2}{*}{1,33} \\
\hline & 5 & 3 & 1 & & \\
\hline \multirow{2}{*}{$\begin{array}{l}\text { Difficultés lors } \\
\text { de la miction }\end{array}$} & $\begin{array}{c}1 \\
\text { jamais }\end{array}$ & $\begin{array}{c}2 \\
\text { parfois }\end{array}$ & $\begin{array}{c}3 \\
\text { souvent }\end{array}$ & \multirow[t]{2}{*}{25} & \multirow[t]{2}{*}{1,32} \\
\hline & 19 & 4 & 2 & & \\
\hline \multirow{2}{*}{$\begin{array}{l}\text { Inconfort avec } \\
\text { certains vêtements }\end{array}$} & OUI & & NON & \multirow{2}{*}{25} & \\
\hline & 9 & & 16 & & \\
\hline
\end{tabular}

$\mathrm{N}=$ Effectifs totaux de répondants

$\bar{M}=$ Valeurs moyennes

Tableau 1 : Perte de sensibilité, problèmes de miction, problèmes d'inconfort dans un échantillon de 25 patients implantés. 
été questionné sur ce point un mois et demi seulement après l'intervention. De fait, si six autres sujets déclarent avoir connu de telles douleurs à l'occasion de la reprise des rapports sexuels, ces douleurs ont, dans leur cas, totalement disparu après trois mois et demi $( \pm 1,5)$ en moyenne.

Les douleurs constitueraient donc une conséquence relativement banale et, en tout cas, passagère de l'intervention . L'optimisme à ce propos gagne encore en constatant que 17 personnes sur 24 estiment n'avoir même jamais été confrontées à ce genre d'inconvénients.

Fait par ailleurs intéressant: seuls les porteurs de modèle gonflable sont ou ont été concernés par des complications de cet ordre.

On répertorie aussi d'autres types d'inconvénients: diminution de la sensibilité du pénis, difficultés lors de la miction, sensation d'inconfort avec certains vêtements. le tableau I résume clairement les données à ce sujet.

\section{Sensibilité du pénis émoussée}

Sept personnes sur vingt-quatre estiment que la sensibilité de leur verge s'est "un peu" émoussée depuis l'intervention; trois jugent cette perte assez "nette". Il convient toutefois de relativiser la portée de l'inconvénient. En effet, quatorze sujets sur vingt-quatre (58\%) estiment leur sensibilité strictement inchangée et, parmi les neuf patients qui se prononcent sur l'impact subjectif de leur perte de sensibilité, cinq, soit plus de la moitié, regardent celle-ci comme "peu gênante". Qui plus est, il semble qu'avec le temps, on puisse parfaitement s'accommoder d'une sensibilité émoussée puisque l'on observe une corrélation inverse (Spearman) de $-0,84 \quad(p=0,001)$ entre l'appréciation subjective de la diminution de sensibilité et la période de follow-up : ces chiffres indiquent que le caractère gênant de ce problème se tempère avec l'habitude .

Par ailleurs, on remarque que les témoignages d'une perte de sensibilité ne se rapportent préférentiellement ni au modèle de prothèse (gonflable ou semi-rigide) ni même au type d'approche chirurgicale (infrapubienne ou péno-scrotale) .

\section{Difficultés lors de la miction}

Les difficultés posées par la prothèse lors de la miction apparaissent plus rarement. Dix-neuf sujets sur vingt-cinq affirment ne jamais connaître ce genre de problèmes. On peut encore se féliciter de n'observer aucune différence en la matière entre les bénéficiaires d'un modèle semirigide et d'un modèle gonflable. A priori, on redoutait que le manque permanent de flaccidité inhérent aux tuteurs semi-rigides puisse poser plus de difficultés aux intéressés; or un seul de ceux-ci déclare vouloir faire les frais de l'installation, chez lui, d'un urinoir. Il faut plutôt mettre les inconvénients relatifs à la miction en rapport avec le manque d'habituation au matériel prothétique: cela vient à l'évidence lorsqu'on découvre une corrélation inverse entre les problèmes liés à la miction et la période de follow-up (coefficient de Spearman=-0,72; $<<0,001$ ). Autrement dit, les patients tendent d'autant moins à faire état de difficultés mictionnelles qu'ils disposent d'un recul important depuis leur opération.

\section{Sensations inconfortables}

Quant aux problèmes d'inconfort avec certains vêtements, on s'étonne à nouveau de ce qu'ils n'apparaissent pas le fait nettement privilégié des porteurs d'un modèle semi-rigide. Nous pensions que le retour à l'état de flaccidité permis par les prothèses gonflables devait éviter ce type d'inconvénients à leurs usagers. Or nos analyses ne donnent lieu à aucune différence significative sur ce plan selon le modèle implanté.

Les vêtements incriminés sont évidemment les maillots et pantalons serrants. Si l'on n'observe aucune corrélation inverse entre la période de follow-up et le fait de relater certains problèmes d'inconfort, on peut cependant logiquement s'attendre à ce qu'avec l'expérience, les sujets deviennent moins enclins à porter des habits occasionnant des sensations inconfortables .

5. Problèmes spécifiques du type de prothèse.

Certains inconvénients se présentent électivement soit aux porteurs de prothèse gonflable, soit

\begin{tabular}{|c|c|c|c|c|c|c|}
\hline \multirow{2}{*}{ PROTHESES GONFLABLES } & \multicolumn{4}{|c|}{ Modalités } & \multirow{2}{*}{$\mathrm{N}$} & \multirow{2}{*}{$\mathrm{M}$} \\
\hline & \multicolumn{4}{|c|}{ Effectifs } & & \\
\hline \multirow{2}{*}{ Difficultés liées au gonflage } & $\begin{array}{c}1 \\
\text { jamais }\end{array}$ & $\begin{array}{c}2 \\
\text { rarement }\end{array}$ & $\begin{array}{c}3 \\
\text { parfois }\end{array}$ & $\begin{array}{c}4 \\
\text { souvent }\end{array}$ & \multirow{2}{*}{17} & \multirow[t]{2}{*}{2} \\
\hline & 8 & 3 & 4 & 2 & & \\
\hline \multirow[t]{2}{*}{ Difficultés liées au dégonflage } & $\begin{array}{c}1 \\
\text { jamais } \\
\end{array}$ & $\begin{array}{c}2 \\
\text { rarement } \\
\end{array}$ & $\begin{array}{c}3 \\
\text { parfois } \\
\end{array}$ & $\begin{array}{c}4 \\
\text { souvent }\end{array}$ & \multirow[t]{2}{*}{17} & \multirow[t]{2}{*}{1,76} \\
\hline & 10 & 3 & 2 & 2 & & \\
\hline \multirow{2}{*}{$\begin{array}{l}\text { Dégonflage non désiré de la } \\
\text { prothèse }\end{array}$} & $\begin{array}{c}1 \\
\text { jamais } \\
\end{array}$ & $\begin{array}{c}2 \\
\text { rarement } \\
\end{array}$ & $\begin{array}{c}3 \\
\text { parfois }\end{array}$ & $\begin{array}{c}4 \\
\text { souvent } \\
\end{array}$ & \multirow[t]{2}{*}{17} & \multirow[t]{2}{*}{1,24} \\
\hline & 14 & 2 & 1 & 0 & & \\
\hline \multirow{2}{*}{$\begin{array}{l}\text { Appréciation subjective des } \\
\text { difficultés liées au } \\
\text { gonflage/dégonflage }\end{array}$} & $\begin{array}{c}1 \\
\text { pas important du tout }\end{array}$ & $\begin{array}{c}2 \\
\text { peu inportant }\end{array}$ & $\begin{array}{c}3 \\
\text { assez important }\end{array}$ & $\begin{array}{c}4 \\
\text { très important }\end{array}$ & \multirow{2}{*}{9} & \multirow{2}{*}{2,4} \\
\hline & 1 & 5 & 1 & 2 & & \\
\hline \multirow{2}{*}{$\begin{array}{l}\text { Difficultés de manipulation } \\
\text { autres que liées au } \\
\text { gonflage/dégonflage }\end{array}$} & $\begin{array}{c}1 \\
\text { jamais }\end{array}$ & $\begin{array}{c}2 \\
\text { rarement } \\
\end{array}$ & $\begin{array}{c}3 \\
\text { parfois } \\
\end{array}$ & $\begin{array}{c}4 \\
\text { souvent } \\
\end{array}$ & \multirow[t]{2}{*}{17} & \multirow[t]{2}{*}{1,65} \\
\hline & 12 & 1 & 2 & 2 & & \\
\hline \multirow{2}{*}{$\begin{array}{l}\text { Appréciation subjective des } \\
\text { difficultés non liées au } \\
\text { gonflage/dégonflage }\end{array}$} & $\begin{array}{c}1 \\
\text { pas important du tout }\end{array}$ & $\begin{array}{c}2 \\
\text { peu important }\end{array}$ & $\begin{array}{c}3 \\
\text { assez important } \\
\end{array}$ & $\begin{array}{c}4 \\
\text { très important } \\
\end{array}$ & \multirow[t]{2}{*}{5} & \multirow{2}{*}{2,8} \\
\hline & 1 & 1 & 1 & 2 & & \\
\hline \multicolumn{7}{|l|}{ PROTHESES SEMI-RIGIDES } \\
\hline \multirow[t]{2}{*}{ Difficultés de manipulation } & $\begin{array}{c}1 \\
\text { jamais } \\
\end{array}$ & $\begin{array}{c}2 \\
\text { rarement } \\
\end{array}$ & $\begin{array}{c}3 \\
\text { parfois } \\
\end{array}$ & $\begin{array}{c}4 \\
\text { souvent } \\
\end{array}$ & \multirow[t]{2}{*}{8} & \multirow[t]{2}{*}{1,62} \\
\hline & 6 & 0 & 1 & 1 & & \\
\hline \multirow{2}{*}{$\begin{array}{l}\text { Appréciation subjective des } \\
\text { difficultés de manipulation }\end{array}$} & $\begin{array}{c}1 \\
\text { pas important du tout }\end{array}$ & $\begin{array}{c}2 \\
\text { peut important }\end{array}$ & $\begin{array}{c}3 \\
\text { assez important }\end{array}$ & $\begin{array}{c}4 \\
\text { très important }\end{array}$ & \multirow{2}{*}{2} & \multirow{2}{*}{3,5} \\
\hline & 0 & 0 & 1 & 1 & & \\
\hline
\end{tabular}

Tableau 2 : Difficultés liées aux modèles gonflables/aux modèles semi-rigides

aux porteurs de tuteurs semi-rigides. Le tableau 2 en donne un aperçu synthétique.

\section{5.a. Problèmes spécifiques rencontrés par les porteurs d'une prothèse gonflable.}

Parmi les usagers d'un modèle gonflable, sept, soit $41 \%$, ne mentionnent actuellement aucun problème relatif au gonflage ou au dégonflage.

Les difficultés d'activation (de gonflage et de dégonflage) sont généralement liées entre elles. Elles tendent aussi à s'estomper avec le temps . De fait, lorsqu'on croise la période de follow-up avec la fréquence des problèmes au gonflage, on observe une corrélation négative (Spearman) de $0,69(\mathrm{p}=0,002)$ et, avec les problèmes de dégonflage, une corrélation de $-0,71(\mathrm{p}=0,001)$. Temps et difficultés à gonfler ou dégonfler la prothèse entretiennent donc des rapports inverses.

Cela témoigne certainement d'un phénomène d'adaptation progressive.

Les dégonflages non souhaités ne surviennent qu'assez rarement.

Les difficultés relatives au gonflage/dégonflage ne portent au surplus guère préjudice à la sexualité; ce excepté pour trois patients qui jugent de tels inconvénients "assez" $(n=1)$ ou "très importants" $(\mathrm{n}=2)$. Elément notable : ce sont également ces trois personnes qui, depuis l'opération, ont le moins de recul (respective- 
ment: un mois et demi, trois mois et demi et quatre mois). En outre, chez l'un d'eux, c'est une cicatrice vicieuse du bas abdomen qui entrave l'expansion de la prothèse et cet inconvénient demeure susceptible d'une intervention correctrice.

Les sujets interrogés un mois et demi et trois mois et demi seulement après l'intervention sont aussi les deux seuls à rencontrer "souvent" des difficultés de manipulation de la prothèse autres que celles liées au gonflage/dégonflage (intromission malaisée) et à trouver ces difficultés "très importantes". Nul doute que ce genre d'inconvénients doive à nouveau être porté au compte d'un manque de familiarisation avec le matériel.

Globalement, trois des dix-sept porteurs de prothèse gonflable $(17,6 \%)$ éprouvent donc des difficultés d'usage considérées comme importantes. Tout porte par ailleurs à croire qu'elles sont amenées à s'atténuer avec le temps.

\section{5.b. Problèmes spécifiques rencontrés par les porteurs d'une prothèse semi-rigide.}

Quant aux porteurs de semi-rigide, deux sur huit (25\%) avouent rencontrer "parfois" ou "souvent" d'importants problèmes de manipulation. Pour l'un, cela s'explique par une rupture de l'armature métallique de la prothèse, rupture rendant la pénétration moins aisée et engendrant l'impression désagréable d'un jeu du tuteur dans la verge: cette prothèse, de type Jonas, a été récemment remplacée par un modèle gonflable (AMS 700). Pour l'autre, c'est un repli du gland lors de l'intromission (que nous nommons le syndrôme du Concorde) qui suscite la plainte: de quelque modèle qu'elle soit, la prothèse ne peut en effet mécaniquement pas entraîner la turgescence du gland, reste que cet implanté-ci est le seul à en déplorer les conséquences.

\section{Discussion}

En somme, si l'on ne peut nier l'existence d'un certain nombre de petits désagréments, il convient d'en relativiser l'impact sur le fonctionnement sexuel. Tout d'abord, les patients estiment volontiers "peu ou pas gênants" les inconvénients dont ils font état. En second lieu, plusieurs de ces problèmes s'atténuent avec l'habitude. Enfin, dans certains cas, il demeure possible d'envisager des aménagements. Les traitements organiques, bien sûr, viennent directe-

\begin{tabular}{|c|c|c|c|c|c|}
\hline \multirow{2}{*}{ ITEM } & \multicolumn{3}{|c|}{ Modalités } & \multirow{2}{*}{$\mathrm{N}$} & \multirow{2}{*}{$\mathrm{M}$} \\
\hline & \multicolumn{3}{|c|}{ Effectifs } & & \\
\hline \multirow{2}{*}{$\begin{array}{l}\text { Satisfaction à propos du volume } \\
\text { et de la longueur procurés à } \\
\text { la verge par la prothèse }\end{array}$} & $\begin{array}{cc}1 & 2 \\
\text { pas important du tout } & \text { peu important }\end{array}$ & $\begin{array}{c}3 \\
\text { assez important }\end{array}$ & $\begin{array}{c}4 \\
\text { très important } \\
\end{array}$ & \multirow[t]{2}{*}{25} & \multirow[t]{2}{*}{2,64} \\
\hline & 5 & 12 & 4 & & \\
\hline
\end{tabular}

Tableau 3 : Problèmes liés aux dimensions de la verge dans un échantillon de 25 patients implantés

\begin{tabular}{|c|c|c|c|}
\hline \multirow[t]{2}{*}{ variables $\mathrm{A}$ à $\mathrm{F}$} & \multicolumn{3}{|c|}{$\begin{array}{l}\text { variables } 1 \text { à } 3 \\
\text { satisfaction exprimée par le patient envers: }\end{array}$} \\
\hline & $\begin{array}{c}1 \\
\text { la qualité de sa vie } \\
\text { sexuelle actuelle } \\
\text { (assistée d'une prothèse) }\end{array}$ & $\begin{array}{c}2 \\
\text { la qualité des } \\
\text { rapports sexuels } \\
\text { permis par la prothèse }\end{array}$ & $\begin{array}{c}3 \\
\text { la qualité de } \\
\text { la prothèse }\end{array}$ \\
\hline $\begin{array}{l}\text { A Fréquence des difficultés } \\
\text { lors de la miction }\end{array}$ & non signif. & $-0,48^{*}$ & non sign. \\
\hline $\begin{array}{l}\text { B Fréquence des difficultés } \\
\text { liées au dégonflage }\end{array}$ & non signif. & $-0,54^{*}$ & $-0,57^{*}$ \\
\hline $\begin{array}{l}\text { C Fréquence des difficultés } \\
\text { liées au gonflage }\end{array}$ & non signif. & $.0,59^{*}$ & non signf. \\
\hline $\begin{array}{l}\text { D Appréciation subjective des } \\
\text { difficultés liées au gonf./dégonf. }\end{array}$ & non signif. & $-0,74^{*}$ & $-0,74$ * \\
\hline $\begin{array}{l}\text { E Appréciation subj. des difficultés } \\
\text { de manipulation non liées au } \\
\text { gonfl. dégonfl. }\end{array}$ & non signif. & non signif. & $-0,88^{*}$ \\
\hline $\begin{array}{l}\text { F Satisfaction envers la taille } \\
\text { du pénis }\end{array}$ & $0,58 * *$ & $0,41^{*}$ & $0,44 *$ \\
\hline
\end{tabular}

$*: \mathrm{p}<0,05-* *: \mathrm{p}<0,01$

Tableau 4 : Corrélations (Spearman) entre les difficultés rencontrées par les patients implantés (variables $\mathrm{A}$ à F) et les indices de satisfaction (variables 1 à 3 )

ment à l'esprit : on songe notamment à la prescription d'analgésiques pour parer aux douleurs. On songe également à une intervention correctrice, par exemple chez la personne qui présente une cicatrice au niveau du bassin, mais peut-être les aménagements devraient-ils aussi se penser sous l'angle d'un encadrement sexologique, un encadrement visant à améliorer et accélérer l'intégration technique et subjective des possibilités offertes par la prothèse. Pour reprendre l'expression d'un de nos patients, il ne serait probablement pas inutile d'organiser "un suivi pratique de remise en fonction". Cette dernière proposition rejoint les conclusions de Coulange (1) à propos de sa propre pratique de l'implantation.

\section{Les dimensions du pénis implanté}

Le problème de la taille de la verge implantée va maintenant nous permettre de pousser davantage encore l'analyse des intrications psychosexologiques dans la chirurgie prothétique.

Ce problème apparaît de façon plus massive et persistante : la satisfaction envers la taille de l'érection permise par la prothèse se montre relativement réduite ( $\mathrm{Cf}$ tableau 3 ) et les plaintes à ce propos ne semblent guère pouvoir s'atténuer avec le temps (corrélation nulle entre les déceptions relatives aux dimensions du pénis implanté et la période de follow-up).

Comme l'indique le tableau 3, seuls quatre sujets se déclarent totalement satisfaits de la taille procurée au pénis par la prothèse. A l'opposé, neuf $(36 \%)$ s'avèrent "peu ou pas du tout satisfaits".

Le tableau 4 nous apprend qu'un bon nombre des problèmes évoqués dans cette étude. interagissent avec les niveaux de satisfaction, niveaux de satisfaction également explorés dans notre enquête de follow-up et exprimés par les sujets sur des échelles en 5 points. La fréquence, I'intensité et le ressenti de certaines difficultés rencontrées par les patients semblent oeuvrer dans le sens d'un tassement des indices de satisfaction.

Dans la mesure où les problèmes liés à la miction, au gonflage/dégonflage et à la manipulation de la prothèse correspondent à un manque de familiarisation au matériel en place, on peut valablement espérer que la relative insatisfaction engendrée par ces inconvénients demeure une donnée transitoire. L'amélioration des indices de satisfaction se révèle, dans pareils cas, logiquement tributaire d'un processus progressif d'adaptation fonctionnelle.

Par contre, dans les cas où l'insatisfaction se rapporte à la taille de la verge, il semble que ce processus d'adaptation fonctionnelle n'entre pas en jeu. On ne doit, sur la base d'un tel lien (échelles de satisfaction - plaintes relatives aux dimensions du pénis), pas s'attendre à un redressement spontané des indices de satisfaction puisque les récriminations envers les dimensions 
de la verge ne s'amendent nullement en fonction du temps. Il devient dès-lors hasardeux de miser sur un processus spontané d'adaptation aux nouvelles dimensions du pénis, un processus en soi susceptible de contribuer à l'amélioration des taux de satisfaction. Réfractaires aux effets de l'habitude, les doléances en rapport à la taille des érections induites par la prothèse foulent notre optimisme au pied. Ces plaintes assombrissent d'autant plus les données qu'elles s'avèrent en relation non seulement avec la satisfaction du sujet lui-même (voir tableau 4) mais également avec celle qu'il attribue à sa compagne à propos de la vie sexuelle du couple (coefficient de Spearman $=0,50 ; p=0,02$ ). L'intensité des plaintes concernant la taille de la verge semble même avoir pour fâcheux corrélat une plus grande occurrence d'éjaculations précoces (coefficient de Spearman $=0,45 ; p=0,029$ ).

\section{Discussion à propos du problème posé par la taille de la verge implantée}

Que ce soit à l'aide d'un modèle gonflable ou semi-rigide, les patients recouvrent bien-sûr rarement une érection comparable à celle d'avant les troubles : il s'agit là d'un phénomène couramment observé. Les candidats à l'implant en sont d'ailleurs avertis.

Néanmoins, la perte en longueur de la verge constitue pour certains l'objet d'une plainte tenace, avec les répercussions que l'on sait (cf supra). La réduction des dimensions de l'organe demeure une cause de déconvenue et, à partir de là, s'annonce susceptible de dénier au vécu érotique une part de sa valeur positive.

Or, s'il faut en croire les études réalisées par Masters et Johnson il semble que, sur le plan de la mécanique sexuelle, la dimension du pénis n'intervient pour ainsi dire pas, sauf cas extrêmes, comme facteur objectif déterminant dans la conduite des rapports. Tout laisse penser qu'une règle d'adaptation aux contingences morphologiques est habituellement de rigueur. Dans cette optique, la qualité des réponses sexuelles dépendrait bien moins des caractéristiques physiques (d'ailleurs extrêmement variables) d'un organisme que de la façon dont elles sont assimilées par la subjectivité des personnes.

Au vu du témoignage de nos sujets, l'adaptation aux modifications des caractères morphologiques de la verge ne va pas de soi pour les prothésés.

Au delà des contraintes physiques qui, toute mesure gardée, peuvent participer à l'explication des plaintes (relevons notamment le cas de deux sujets pour lesquels le défaut de taille se rapporte principalement à des difficultés d'activation du matériel gonflable), il faut vraisemblablement compter avec certaines difficultés d'intégration psychosexologique d'une nouvelle donne érectile.

Pour quantité de patients, de couples, l'appréciation de la sexualité permise par la prothèse se réfère à la situation antérieure aux troubles de l'érection. Cette tendance larvée à la comparaison peut, on s'en doute, venir altérer les capacités de s'épanouir érotiquement sur la base d'un organe inévitablement raccourci par rapport aux circonstances naturelles de l'érection.

En outre, si l'expérience de l'impuissance ne va pas sans affecter une certaine image de la virilité, nos données nous apprennent également que la solution prothétique laisse subsister des blessures narcissiques (2). Nul besoin d'une maitrise approfondie des notions psychodynamiques pour avoir à l'esprit la place essentielle tenue par l'érection dans la symbolique phallique. Les grivoiseries communes suffisent du reste à nous convaincre de l'empire de l'association "virilité=pénis imposant". Ainsi, devoir son érection symbole de puissance mâle- à un agent extérieur ne doit déjà précisément pas suffire à redorer le blason masculin. On imagine sans peine comment pareil sentiment d'insuffisance virile se renforce, se cristallise dans la mesure où cette blessante idée d'une érection désormais dépendante de l'artifice prothétique se matérialise de surcroît par une puissance érectile réduite comparativement à l'époque d'avant les troubles.

Il n'est donc guère surprenant de constater l'ampleur prise par les frustrations relatives aux dimensions du pénis, ni même de constater que celles-ci puissent quelque peu déstabiliser les relations d'un couple à l'érotisme.

Pour surmonter ces inconvénients, une prise en charge psychosexologique représente à l'évidence un outil privilégié.

\section{CONCLUSION}

Pour la plupart des difficultés relatées par les patients implantés (douleurs, problèmes de miction, d'inconfort, de sensibilité de la verge et de manipulation de la prothèse), il n'y a pas lieu de dramatiser. De manière générale, les sujets tendent à en minimiser l'impact subjectif . Si, dans certains cas, une aide médicale peut être apportée, la résolution de ces inconvénients emprunte surtout les voies spontanées de l'adaptation progressive au matériel prothétique en place. Reste qu'un suivi sexologique pourrait éventuellement faciliter et accélérer le processus.

La situation se révèle plus délicate lorsqu'on a affaire à des doléances incriminant les dimensions de l'érection permise par la prothèse. Les plaintes à ce propos apparaissent relativement fréquentes, non enrayables par une adaptation spontanée et, à partir de là, elles semblent capables d'affecter en continu la qualité du vécu sexuel. Etant donné les très probables fondements psychologiques de ce genre de plaintes, on soulignera évidemment la pertinence d'un accompagnement psycho- ou sexothérapeutique.

\section{REFERENCES}

1 - C. Coulange, B. Soler, M. Rampal : Les prothèses dans le traitement de l'impuissance. Sexologies, vol. 1, n 2. 1991.

2 - P. Kempeneers, R. Andrianne, C. Mormont : La prothèse pénienne : contrariétés d'une virilité artificielle. A paraitre.

3- W.E. Masters, V.E. Johnson : Human sexual response. Little Brown. Boston, 1966.

RESUME : Vingt-cinq patients implantés entre 1982 et 1991 ont répondu à un questionnaire de follow-up. Certains y rapportent des problèmes de douleurs, des difficultés liées à la miction, à une perte de sensibilité de la verge, des sensations inconfortables et des difficultés de manipulation de la prothèse. Beaucoup de ces inconvénients sont toutefois minimisés par les patients concernés. La plupart de ces difficultés semblent du reste appelées à s'estomper progressivement avec le temps. Un processus spontané d'adaptation fonctionnelle est ici à l'oeuvre. Un problème plus délicat existe à propos des dimensions procurées au pénis par la prothèse: fréquentes et persistantes, les plaintes à ce sujet sont peut-être en partie psychologiquement déterminées. Pour réduire ce type de difficultés autant que pour faciliter l'adaptation fonctionnelle au matériel en place, un accompagnement psychosexologique des patients implantés s'avérerait probablement une option judicieuse et souhaitable. Mots-clés : Impuissance - Prothèse pénienne. Andrologie, 1992, 2 : 130-133 